\title{
MGMT Promoter Methylation Testing Was Performed
}

National Cancer Institute

\section{Source}

National Cancer Institute. MGMT Promoter Methylation Testing Was Performed. NCI

Thesaurus. Code C160407.

An indication that MGMT promoter methylation analysis was performed during the study. 